\title{
Vulnerability to Depression: From Brain Neuroplasticity to Identification of Biomarkers
}

\author{
Aurélie Blugeot, ${ }^{1,2,3 \star}$ Cyril Rivat, ${ }^{1,2,3 \star}$ Elodie Bouvier, ${ }^{1,2,3}$ Jenny Molet, ${ }^{1,2.3}$ Amandine Mouchard, ${ }^{1,2,3}$ Brigitte Zeau, ${ }^{1,2,3}$ \\ Christophe Bernard, ${ }^{4}$ Jean-Jacques Benoliel, ${ }^{1,2,3,5}$ and Chrystel Becker ${ }^{1,2,3,6}$ \\ ${ }^{1}$ Université Pierre et Marie Curie-Paris 6, UMRS 975, Pain Team, Site Pitié-Salpêtrière, Paris 75013, France, ${ }^{2}$ INSERM, U 975, Paris 75013, France, ${ }^{3}$ CNRS, \\ UMR 7225, Paris 75013, France, ${ }^{4}$ INSERM, U 751, Marseille 13385, France, ${ }^{5}$ Service de Biochimie Endocrinienne et Oncologique, Hôpital de la Pitié- \\ Salpêtrière, Paris 75013, France, and ' Université Paris Descartes, Sorbonne Paris Cité, Faculté de Médecine, Paris 75006, France
}

A stressful event increases the risk of developing depression later in life, but the possible predisposing factors remain unknown. Our study aims to characterize latent vulnerability traits underlying the development of depressive disorders in adult animals. Four weeks after a priming stressful event, serum corticosterone concentration returned to control values in all animals, whereas the other biological parameters returned to basal level in only $58 \%$ of animals (called nonvulnerable). In contrast, $42 \%$ of animals displayed persistent decreased serum and hippocampus BDNF concentrations, reduced hippocampal volume and neurogenesis, CA 3 dendritic retraction and decrease in spine density, as well as amygdala neuron hypertrophy, constituting latent vulnerability traits to depression. In this group, called vulnerable, a subsequent mild stress evoked a rise of serum corticosterone levels and a "depressive" phenotype, in contrast to nonvulnerable animals. Intracerebroventricular administration of 7,8-dihydroxyflavone, a selective TrkB receptor agonist, dampened the development of the "depressive" phenotype. Our results thus characterize the presence of latent vulnerability traits that underlie the emergence of depression and identify the association of low BDNF with normal corticosterone serum concentrations as a predictive biomarker of vulnerability to depression.

\section{Introduction}

Despite the prevalence of depression and its considerable impact in public health, its pathophysiological mechanisms remain poorly understood (Krishnan and Nestler, 2008). Epidemiological, clinical, and basic research studies have revealed the existence of a complex interplay between genetic and environmental elements, with the physiological and psychological history of the subject playing a particularly important role (McEwen and Stellar, 1993; Cryan and Slattery, 2007; Jabbi et al., 2007; Jacobson and Cryan, 2007). Knowledge of the risk factors contributing to susceptibility to stress and of the mechanisms by which they act in the subsequent development of depression is crucial (Brown et al., 1987; Post, 1992; Kessler, 1997; de Kloet et al., 2005). Sustained or repetitive stress in adulthood may trigger maladaptive changes in some individuals, producing a vulnerable phenotype and may act as a trigger for mechanisms that leave predisposed individuals at increased risk of illness development (de Kloet et al., 2005). Thus, psychological stressful events (e.g., death of rel-

\footnotetext{
Received March 15, 2011; revised; ; accepted July 11, 2011.

Author contributions: J.-J.B. and C. Becker designed research; A.B., C.R., E.B., J.M., A.M., B.Z., and C. Becker performed research; A.B., C.R., E.B., J.M., C. Bernard, J.-J.B., and C. Becker analyzed data; C.R., C. Bernard, J.-J.B., and C. Becker wrote the paper.

This work was supported by grants from the Institut National de la Santé et de la Recherche Médicale and Université Pierre et Marie Curie and fellowships from the Fondation pour la Recherche Médicale and Medicen. We thank J.-C. Lambert, P. V. Piazza, and M. Pohl for critical reading of this manuscript.

${ }^{*}$ A.B. and C.R. contributed equally to this work.

The authors declare no competing financial interests.

Correspondence should be addressed to Chrystel Becker at the above address. E-mail: christel.becker@upmc.fr. DOI:10.1523/JNEUROSCI.1309-11.2011

Copyright $\odot 2011$ the authors $\quad 0270-6474 / 11 / 3112889-11 \$ 15.00 / 0$
}

atives, divorce, humiliation or defeat, deterioration of financial status) may sensitize neurobiological systems, leading to a state of vulnerability to the subsequent development of depression later in life. However, biological markers corresponding to residual traces of past stressful episodes and predictive to depression sensitivity remain to be identified.

We hypothesized that some alterations described in depressive patients, such as decrease in hippocampal volume and BDNF serum concentrations (Sheline et al., 1999; Bremner et al., 2000; Shimizu et al., 2003; Karege et al., 2005; Czeh and Lucassen, 2007; Kim et al., 2007; Pittenger and Duman, 2008; Schmidt and Duman, 2010), could occur before the depressive state, thus constituting predictive markers of depression sensitivity. To test this hypothesis we designed a sensitization paradigm (SSP), in which adult animals were subjected to a first intense stress using social defeat (Miczek et al., 2008), which induces a sixfold increase in serum corticosterone concentration (Becker et al., 2001; Andre et al., 2005), followed 4 weeks later by a second stress [chronic mild stress (CMS)]. Neuroanatomical and biochemical parameters were measured during the SSP, and the depression-like profile was estimated. The main goal of this longitudinal study was to characterize residual traces evoked by the first intense stress to predict which animals would develop "depression" following CMS.

\section{Materials and Methods \\ Animals}

Male Sprague Dawley rats from the same breeder (Centre d'Elevage R. Janvier) and weighing 324 to $349 \mathrm{~g}$ were used as intruder rats. On their arrival at the laboratory, they were housed in chronobiologic animal 
facilities (Enceinte Autonome d'Animalerie; A110SP, Thermo Electron). The chronobiologic facility is equipped with regularly spaced, sound-proof, temperature-controlled compartments, each supplied with filtered air. Each compartment has its own light/dark cycle control. Sprague Dawley rats were housed together for $4 \mathrm{~d}$ and were then transferred to individual cages (length, $45 \mathrm{~cm}$; width, $25 \mathrm{~cm}$; height, 17 cm) $14 \mathrm{~d}$ before the start of the experiments (see Fig. 1a). Long Evans rats (Centre d'Elevage R. Janvier) weighing 700 to $800 \mathrm{~g}$ were used as resident rats in confrontation encounters. All animals were kept under controlled environmental conditions $\left(22 \pm 1^{\circ} \mathrm{C}, 60 \%\right.$ relative humidity, $12 \mathrm{~h}$ light/dark cycle with lights on at 7:00 A.M., food and water ad libitum). Procedures involving animals and their care were performed in accordance with institutional guidelines conforming to national and international laws and policies (council directive \#87-848, October 19, 1987, Ministère de l'Agriculture et de la Forêt, Service Vétérinaire de la Santé et de la Protection Animale, permissions \#75-1178 to J.J.B.).

All measurements were performed by individuals blind to the treatment received by the rats.

\section{Social-defeat procedure}

The social-defeat procedure was performed as described previously (Becker et al., 2001; Andre et al., 2005). Briefly, the procedure involved subjecting the same pairs of residents and intruders to four daily conditioning sessions [from Day 0 (D0) to D4]. The $45 \mathrm{~min}$ conditioning sessions were divided into two consecutive periods. During Period 1 (30 min), intruders were placed individually in a protective cage inside the resident animal's home cage. The protective cage allowed unrestricted visual, auditory, and olfactory contacts with the resident but prevented close physical contact. During Period 2 (15 min), the protective cage was removed, either with the resident remaining present, allowing physical confrontation with the intruder [three to four confrontations of $\sim 10 \mathrm{~s}$, during each of which the intruding [defeated (DF)] animal was always dominated by the resident rat] or with the resident removed, giving the intruder access to the entire resident home cage [control (C) intruders]. The control intruders were therefore never physically attacked and defeated by the resident.

\section{Chronic mild stress protocol}

The CMS protocol was derived from that described by Willner et al. (1992). Briefly, various mild stresses were applied every day, over a period of 3 weeks. Briefly, from Monday to Friday, every morning, animals were placed in a small cage $(10 \times 16 \times 15 \mathrm{~cm})$ for $1 \mathrm{~h}$. In the afternoon, they were shaken for $10 \mathrm{~min}$ (Monday and Thursday), placed in a small cage containing water ( $2 \mathrm{~cm}$ high; Tuesday) or placed in another cage for $4 \mathrm{~h}$ (Wednesday and Friday). Every night, the cage was inclined $\left(45^{\circ}\right.$; Tuesday and Thursday) or contained wet bedding (Wednesday and Friday). On Saturday and Sunday, the rats were subjected to a reversed dark/light cycle for $30 \mathrm{~min}$, every $3 \mathrm{~h}$.

\section{SSP}

Sprague Dawley animals were subjected to chronic social defeat (CSD) and then, 4 weeks later, to chronic mild stress (SSP rats) (see Fig. 1a). In some experiments, control intruders (without social defeat) were submitted to the 3 weeks of CMS 4 weeks later (CMS rats).
Table 1. Statistical analyses of the CMS effects on biological, behavioral, and neuroanatomical parameters

\begin{tabular}{ll}
\hline & CMS effect \\
\hline Corticosterone levels & $F_{(1,22)}=7.66, p=0.01$ \\
Adrenal gland weight & $F_{(1,22)}=0.002, p=0.96$ \\
Serum BDNF levels & $F_{(1,22)}=0.021, p=0.88$ \\
Hippocampal BDNF levels & $F_{(1,9)}=0.11, p=0.73$ \\
Immobility time & $F_{(1,12)}=0.026, p=0.87$ \\
Sweet water consumption & $F_{(1,14)}=3.6, p=0.08$ \\
Hippocampal volume & $F_{(1,6)}=0.54, p=0.48$ \\
Cell proliferation & $F_{(1,6)}=0.004, p=0.95$ \\
Apical dendrite length & $F_{(1,18)}=0.32, p=0.57$ \\
Basal dendrite length & $F_{(1,18)}=0.51, p=0.48$ \\
Dendritic spines & $F_{(1,72)}=0.55, p=0.45$ \\
Dendrite length of amygdala neurons & $F_{(1,8)}=1.53, p=0.25$ \\
\hline
\end{tabular}

Statistical analyses of the effects of CMS on corticosterone levels, adrenal gland weight, serum and hippocampal BDNF concentrations, hippocampal volume, cell proliferation, apical and basal CA3 dendrite lengths, hippocampal CA3 dendritic spine, dendrite length of amygdala neurons, sweet water consumption, and immobility time based on one-way ANOVA.

Control rats corresponded to control intruders not exposed to the 3 weeks of CMS (C rats).

\section{Forced-swimming test}

At D9, D35, or D57, forced-swimming test (FST) experiments were performed between 9:30 A.M. and 1:00 P.M. Experiments were performed as 


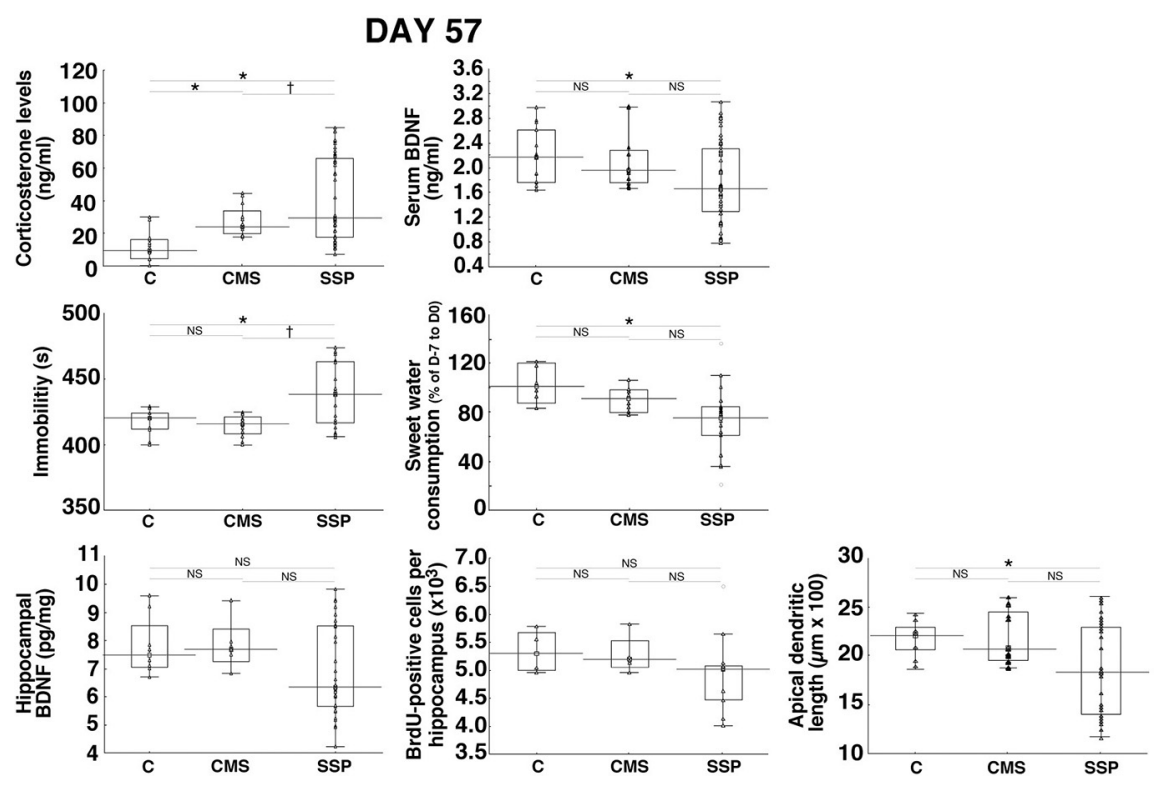

Figure 2. Sensitization procedure induces interindividual variability. At D57, serum corticosterone and BDNF concentrations, immobility time in the FST, sweet water consumption, hippocampal BDNF concentrations, number of BrdU-positive hippocampal cells, and apical dendrite length of CA3 hippocampal neurons for animals subjected to the SSP $(n=9-48)$, control rats (animals with no history of social defeat and CMS, $n=4-15)$, and CMS rats $(n=4-12)$ are shown. Data presented as box plots show the 25 th and 75 th percentiles. The bars in the boxes represent the medians. Circles represent atypical values. ${ }^{*} p<0.05$ versus $C$ rats; ${ }^{\dagger} p<0.05$ versus CMS rats.

described by Becker et al. (2008). Rats were placed individually into glass cylinders ( $40 \mathrm{~cm}$ high; $20 \mathrm{~cm}$ diameter) containing $29 \mathrm{~cm}$ of water at $30 \pm$ $1^{\circ} \mathrm{C}$. After $8 \mathrm{~min}$, they were transferred to a $30^{\circ} \mathrm{C}$ drying environment for $15 \mathrm{~min}$. The water was changed after each animal's session and the cylinder cleaned to avoid the influence of alarm pheromones left behind by the previous animal. The immobility time was measured with a stopwatch. A rat was considered immobile when floating and making only the necessary movements to keep its nostrils above the water surface. A trained experimenter blind to the treatment made animal observations and measurements. Both room and water temperatures were monitored at the end of each session. Each rat was subjected individually to only one swimming session.

\section{Sweet water consumption}

Experiments were performed as described by Becker et al. (2008). From 1 week before the beginning of the conditioning sessions (control period, Day -7 to Day 0), two bottles (one filled with water and the other one containing $1.5 \%$ sucrose) were continuously available to rats. Sucrose and water intakes were measured daily at 9:00 A.M. Bottles were switched every day from left to right side of the cage throughout the experiment to avoid any place preference. Weekly sweet water consumption was expressed as a percentage of control values (Day -7 to Day 0 ). Sweet water consumption corresponds to sucrose preference. Indeed, during the control period (Day -7 to Day 0 ), rats drank significantly more sweet water $(\approx 30 \mathrm{ml} / \mathrm{d})$ than plain water $(\approx 15 \mathrm{ml} / \mathrm{d})$, showing a preference for the sweet water. When a decrease of sweet water consumption was observed, the plain water intake was unchanged $(\approx 15 \mathrm{ml} / \mathrm{d})$.

\section{Activity of the hypothalamic-pituitary-adrenal axis}

When the rats were killed (D9, D35, or D57), blood from trunk vessels was collected into chilled tubes and corticosterone was quantified by RIA (ICN Biomedicals) using $\left[{ }^{125} \mathrm{I}\right]$ corticosterone as a radiotracer (Andre et al., 2005). Adrenal glands were removed, dissected free of adhering fat, and weighed. Organ weights are expressed relative to body weights (milligram of gland per $100 \mathrm{~g}$ body weight). Rats were decapitated at midday in a quiet separate room, one by one, with the bench cleaned between rats.

\section{Serum BDNF assay}

Blood samples $(200 \mu \mathrm{l})$ of awake rats were collected at different time points (D9, D35, D57 at midday) from the vein of the tail into Eppendorf tubes. After centrifugation, serum was separated and stored at $-20^{\circ} \mathrm{C}$ until analysis for BDNF. BDNF concentrations were determined at dilution of 1:25 with a commercial BDNF assay (Promega), in 96-well plates (Corning Costar EIA plate), according to the manufacturer's instructions.

\section{Hippocampal BDNF}

Extraction. When the rats were killed (D9, D35, or D57), the brains were rapidly removed. Bilateral hippocampi were rapidly dissected and stored at $-80^{\circ} \mathrm{C}$. At the time of analysis, samples were weighed, and BDNF was extracted as described by Szapacs et al. (2004). Two milliliters of lysis buffer (100 mM PIPES, $500 \mathrm{~mm}$ $\mathrm{NaCl}, 0.2 \%$ Triton $\mathrm{X}-100,0.1 \% \mathrm{NaN}_{3}, 2 \%$ $\mathrm{BSA}$, and $2 \mathrm{~mm}$ EDTA) containing freshly prepared protease inhibitors (200 $\mu \mathrm{M}$ PMSF, 0.3 $\mu \mathrm{M}$ aprotinin, and $10 \mu \mathrm{M}$ leupeptin) were added to each sample. Samples were then sonicated by pulses at $1 \mathrm{~s}$ intervals for $15 \mathrm{~s}$. An additional $1 \mathrm{ml}$ of lysis buffer was added, and the samples were resonicated. All homogenates were centrifuged at $16,000 \times g$ for $30 \mathrm{~min}$ at $4^{\circ} \mathrm{C}$, and supernatants were removed and frozen at $-20^{\circ} \mathrm{C}$ until assay.

Assay. BDNF concentrations were determined at dilution of 1:10 with the commercial

BDNF assay described above.

\section{Cell proliferation and hippocampal volume}

At D9, D35, or D57, rats received two injections of BrdU (75 mg/kg, i.p; Sigma) at $2 \mathrm{~h}$ intervals. Twenty-four hours after the first BrdU injection, animals were anesthetized intraperitoneally with $375 \mathrm{mg} / \mathrm{kg}$ chloral hydrate. Transcardiac perfusion with $4 \%$ cold paraformaldehyde (PFA) in PBS was performed. After perfusion, brains were postfixed by overnight incubation in $4 \% \mathrm{PFA} / \mathrm{PBS}$ at $4^{\circ} \mathrm{C}$. The next day, serial sections $(40 \mu \mathrm{m}$ thick) were cut through the entire hippocampus (plates 26-40) on a freezing microtome, and sections were stored overnight in $\mathrm{PBS}$ at $4^{\circ} \mathrm{C}$. Immunochemistry and volumetric analysis of the granule cell layer (GCL) were performed as described previously (Becker et al., 2008). All BrdU-labeled cells in the dentate gyrus and hilus were counted by an experimenter blind to the slide code. The total number of BrdU-labeled cells per dentate gyrus and the volumetric analysis of the GCL were determined using AxioVision 4.7 software and a Zeiss Imager M1 microscope.

\section{Golgi staining}

At D9, D35, or D57, unperfused brains were rapidly removed and processed with the FD Rapid Golgistain kit (FD NeuroTechnologies). For correct analysis, Golgi-impregnated CA3 hippocampal or amygdala neurons had to satisfy the following criteria: (1) the cell body and dendrites had to be fully impregnated, (2) the neuron had to be relatively isolated from surrounding neurons, (3) the cell had to be located in the CA3 region of the hippocampus or in the central amygdala, and (4) there had to be full-length dendrites present. Histological quantification, involving the measurement of CA3 or amygdala dendritric branch length for selected neurons, was performed with AxioVision 4.7 software and a Zeiss Imager M1 microscope. For each rat, total dendrite length was measured for amygdala neuron and separately for the apical branch and the basal sections of the CA3 neuron, as described by McLaughlin et al. (2005) and Conrad et al. (2007). We selected three to five hippocampal neurons per rat for measurement by an investigator blind to the origin of the experimental animal. Amygdala and CA3 dendritic remodeling analyses were performed on the same animals. 
Table 2. Statistical analyses of the stress effects on biological, behavioral, and neuroanatomical parameters

\begin{tabular}{|c|c|}
\hline & Stress effect \\
\hline \multicolumn{2}{|l|}{ Serum BDNF levels } \\
\hline D9 & $F_{(1,28)}=44.78, p<0.0001$ \\
\hline D35 & $F_{(1,47)}=4.00, p=0.05$ \\
\hline D57 & $F_{(2,72)}=3.65, p=0.043 ; F_{(2,60)}=40.71, p<0.0001$ \\
\hline D35 of SSP rats & $F_{(2,60)}=37.29, p<0.0001$ \\
\hline \multicolumn{2}{|c|}{ Corticosterone levels } \\
\hline D9 & $F_{(1,28)}=101.98, p<0.0001$ \\
\hline D35 & $F_{(1,47)}=0.006, p=0.93$ \\
\hline D57 & $F_{(2,72)}=11.05, p<0.0001 ; F_{(2,60)}=243.76, p<0.000$ \\
\hline \multicolumn{2}{|c|}{ Adrenal gland weight } \\
\hline D9 & $F_{(1,28)}=28.12, p<0.0001$ \\
\hline D35 & $F_{(1,47)}=2.70, p=0.10$ \\
\hline D57 & $F_{(2,60)}=42.00, p<0.0001$ \\
\hline \multicolumn{2}{|c|}{ Hippocampal BDNF levels } \\
\hline D9 & $F_{(1,10)}=16.93, p=0.0021$ \\
\hline D35 & $F_{(2,19)}=21.16, p<0.0001$ \\
\hline D57 & $F_{(2,41)}=1.35, p=0.26 ; F_{(2,35)}=19.54, p<0.0001$ \\
\hline \multicolumn{2}{|r|}{ (2,41) (2) } \\
\hline D9 & $F_{(1,8)}=34.45, p=0.0004$ \\
\hline D35 & $F_{(2,12)}^{(1,0)}=5.55, p=0.019$ \\
\hline D57 & $F_{(2,14)}^{(2,12)}=0.47, p=0.63 ; F_{(2,10)}=17.28, p=0.0006$ \\
\hline \multicolumn{2}{|c|}{ Hippocampal volume } \\
\hline D9 & $F_{(1,8)}=20.92, p=0.0018$ \\
\hline D35 & $F_{(2,12)}=2.19, p=0.15$ \\
\hline D57 & $F_{(2,10)}=5.50, p=0.02$ \\
\hline \multicolumn{2}{|c|}{ Apical dendrite length } \\
\hline D9 & $F_{(1,30)}=41.84, p<0.0001$ \\
\hline D35 & $F_{(2,45)}=27.88, p<0.0001$ \\
\hline D57 & $F_{(2,57)}=4.54, p=0.01 ; F_{(2,45)}=43.15, p<0.0001$ \\
\hline \multicolumn{2}{|r|}{ (2,5) } \\
\hline D9 & $F_{(1,95)}=21.76, p<0.0001$ \\
\hline D35 & $F_{(2,186)}=4.46, p=0.003$ \\
\hline D57 & $F_{(2,161)}^{(2,100)}=9.97, p<0.0001$ \\
\hline \multicolumn{2}{|c|}{$\begin{array}{l}\text { Dendritic length of amygdala } \\
\text { neurons }\end{array}$} \\
\hline D9 & $F_{(1,14)}=26.63, p<0.0001$ \\
\hline D35 & $F_{(2,21)}=11.56, p=0.0004$ \\
\hline D57 & $F_{(2,21)}=11.43, p=0.0004$ \\
\hline \multicolumn{2}{|l|}{ Immobility time } \\
\hline D9 & $F_{(1,16)}=0.69, p=0.42$ \\
\hline D35 & $F_{(2,24)}=0.95, p=0.41$ \\
\hline D57 & $F_{(2,40)}=8.01, p=0.001 ; F_{(2,28)}=20.71, p<0.0001$ \\
\hline \multicolumn{2}{|c|}{ Sweet water consumption } \\
\hline D35 & $F_{(2,23)}=0.34, p=0.71$ \\
\hline D57 & $F_{(2,31)}=5.27, p=0.01 ; F_{(2,23)}=20.51, p<0.0001$ \\
\hline
\end{tabular}

Statistical analyses of the stress effects on serum BDNF levels, corticosterone concentration, adrenal gland weight, hippocampal BDNF concentration, cell proliferation, hippocampal volume, apical dendrite lengths of CA3 neurons, hippocampal CA3 dendritic spine, dendrite length of amygdala neurons, immobility time, and sweet water consumption based on one-way ANOVA.

Dendritic tracing was quantified by Sholl (1953) analysis. A transparent grid with concentric rings, $20 \mu \mathrm{m}$ apart, was placed over the dendritic drawing so that the center of the circles coincided with the center of the cell body. The number of intersections between the dendrites and the concentric circles was counted and plotted as a function of the distance from the soma. The density of dendritic spines was estimated by counting the number of apical dendritic spines in three to four segments of $15 \mu \mathrm{m}$ located in the stratum radiatum at a $1000 \times$ resolution. Spine density was expressed as the number of spines per $15 \mu \mathrm{m}$ of apical dendrite length.

\section{Drugs and treatments}

At D35, blood samples $(200 \mu \mathrm{l})$ of awake rats were collected from the vein of the tail for later BDNF concentration determination allowing the classification in vulnerable $(\mathrm{V})$ or nonvulnerable $(\mathrm{NV})$ animals. Then, rats anesthetized with isofluorane were placed in a stereotaxic apparatus

\section{a $\quad$ SSP (D57)}

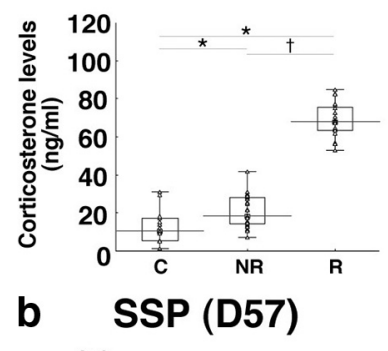
Adrenal gland weight:
C: $10.07 \pm 0.40 \mathrm{mg} / 100 \mathrm{~g}$
$\mathrm{NR}: 9.28 \pm 0.23 \mathrm{mg} / 100 \mathrm{~g}$
R: $\quad 12.59 \pm 0.19 \mathrm{mg} / 100 \mathrm{~g}$ *†
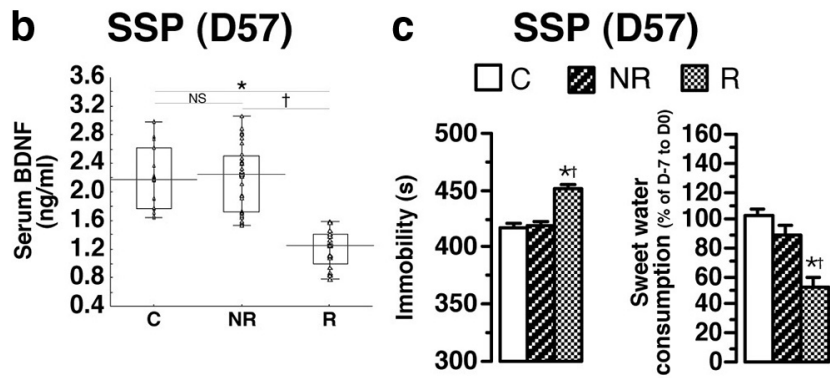

Figure 3. Segregation of rats submitted to the SSP into responder (depressive) and nonresponder (nondepressive) animal populations. $\boldsymbol{a}$, Based on the corticosterone concentrations measured in CMS rats, the highest corticosterone level $(43.94 \mathrm{ng} / \mathrm{ml})$ was set as a cutoff for splitting the SSP animal population into two subpopulations: those with values of $>43.94 \mathrm{ng} / \mathrm{ml}$, named responder rats $(n=20)$ and those with values of $<43.94$ $\mathrm{ng} / \mathrm{ml}$, called nonresponder rats $(n=28)$. $\boldsymbol{b}$, Distinct serum BDNF concentrations between $R, N R$, and $C(n=15)$ animals. c, Evaluation of depression-like profile by the FST and the sweet water consumption of $R$, NR, and $C$ animals $(n=8-12)$. Data presented as box plots show the 25th and 75th percentiles. The bars in the boxes represent the medians. ${ }^{*} p<0.05$ versus $C$ rats; ${ }^{\dagger} p<0.05$ versus NR rats.

(Kopf Instruments). A 26-gauge stainless-steel cannula was implanted just above the roof of the right lateral ventricle (stereotaxic coordinates with respect to bregma: $1 \mathrm{~mm}$ caudal and $1.5 \mathrm{~mm}$ lateral; Paxinos and Watson, 1986) and was lowered $4 \mathrm{~mm}$ below the surface of the skull. The cannula was anchored to the skull by using acrylic dental cement. The cannula was connected to Alzet osmotic minipump (Model 2004; Charles River Laboratories) implanted under the skin of the back. Osmotic pumps were fulfilled with a selective TrkB receptor agonist 7,8dihydroxyflavone (7,8-DHF; $240 \mathrm{ng} / \mathrm{d}$; Tokyo Chemical) (Jang et al., 2010 ) or vehicle. 7,8 -DHF was dissolved in sterile $0.9 \%$ saline adjusted to pH 7 with $0.1 \mathrm{~m} \mathrm{NaOH}$. 7,8-DHF or vehicle was delivered continuously from D35 until the end of the experiment (D57).

A typical tricyclic antidepressant compound (imipramine, $16 \mathrm{mg} /$ $\mathrm{kg} / \mathrm{d}$; ICN) was dissolved in distilled water and used to fill ALZET osmotic mini-pumps (Model 2002; Charles River Laboratories). Imipramine- or distilled water-filled pumps were implanted subcutaneously on the backs of intruder rats under light anesthesia with isofluorane the day after completion of the social-defeat procedure (D5) and were removed at D35.

\section{Statistical analyses}

Box plots, discriminant analysis, classification matrix analysis, and cluster analysis were performed with Statistica (version 7.1). Differences in adrenal gland weight, corticosterone levels, hippocampal cell proliferation, lengths of the dendrites of CA3 hippocampal neurons, lengths of the dendrites of amygdala neurons, dendritic spines, hippocampal or serum BDNF concentrations, sweet water consumption, and immobility time (FST) were validated by one-way ANOVA. The group effect and treatment effect were validated by two-way ANOVAs. All data are presented as means \pm SEM. When ANOVA revealed a significant effect, a post hoc Bonferroni test was performed to determine whether differences were truly statistically significant.

\section{Results}

Effects of 3 week CMS protocol on biochemical, behavioral, and neuroanatomical parameters

Several groups of animals were used. Experimental animals were submitted to CSD, followed by CMS 4 weeks later (Fig. 1a). CMS 
a

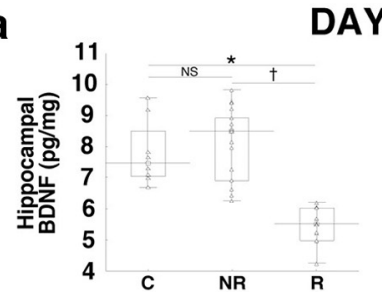

b

Hippocampal volume: C: $10.25 \pm 0.32 \mathrm{~mm}^{3}$ NR: $10.08 \pm 0.12 \mathrm{~mm}^{3}$ $\mathrm{R}: \quad 8.41 \pm 0.61 \mathrm{~mm}^{3}$ *
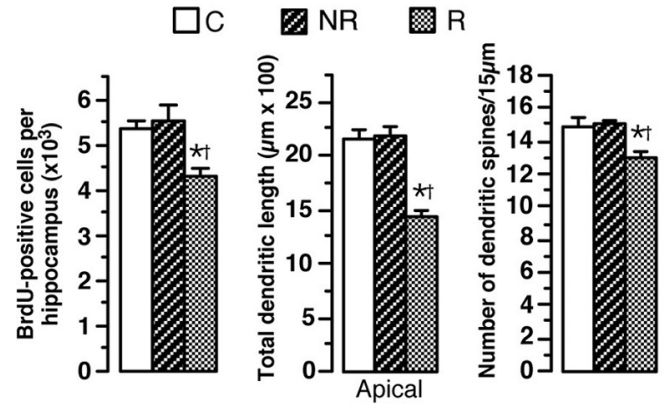

3

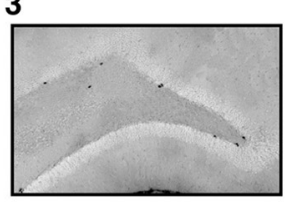

2
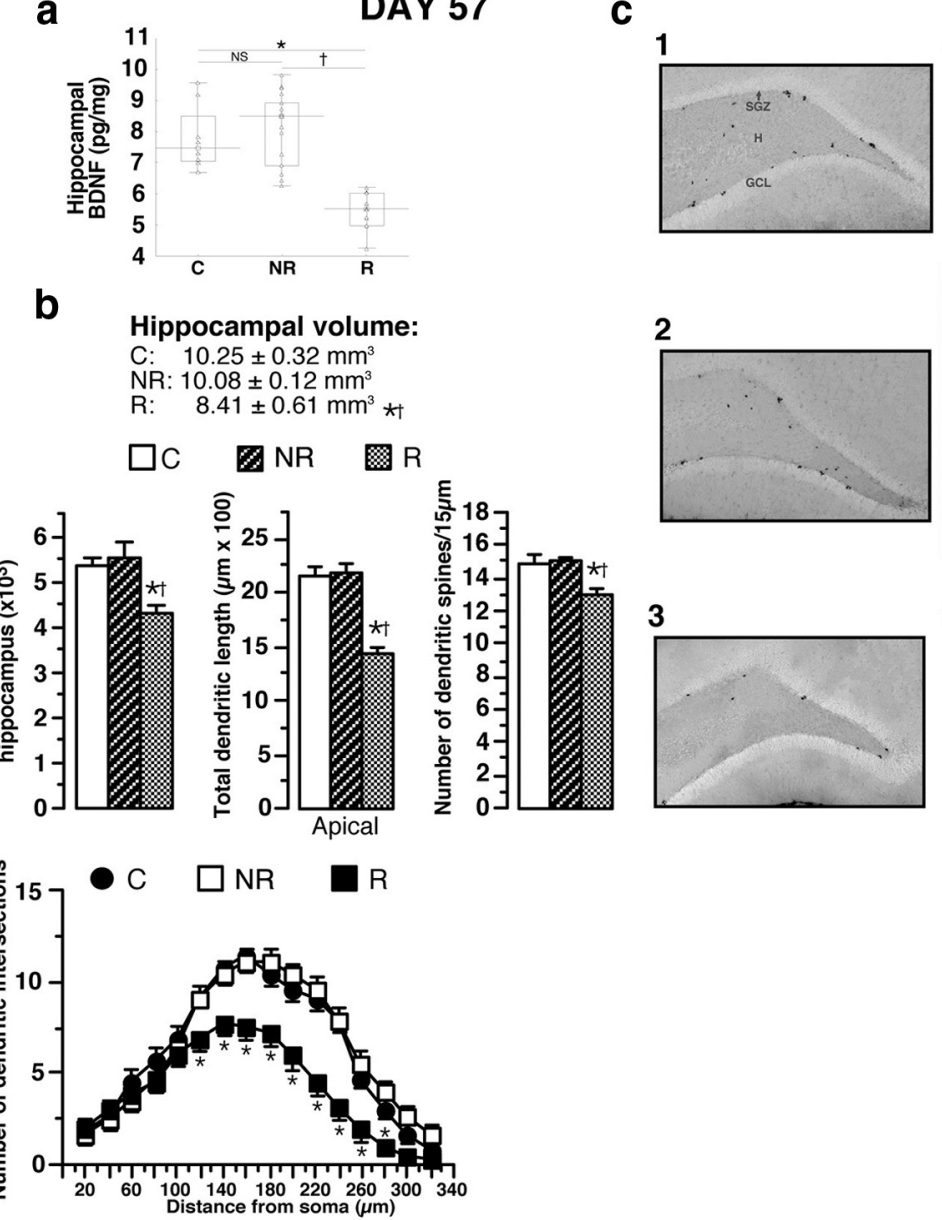

Figure 4. Responder (depressive) and nonresponder (nondepressive) animal populations displayed distinct neuroanatomical alterations at the end of the SSP. $\boldsymbol{a}$, At D57, hippocampal BDNF concentrations in responder animals ( $n=12)$, nonresponder ( $n=$ 18 ), and control rats $(n=8) \cdot \boldsymbol{b}$, The low hippocampal BDNF concentrations in R animals were associated with a lower hippocampal volume, a smaller number of BrdU-positive hippocampal cells, a retraction of apical CA3 hippocampal dendrites, and a decrease of dendritic spines. Sholl analysis for C, NR, and R animals is shown. C, Representative BrdU-labeled sections of the dentate gyrus (1, 2, 3; $50 \times$ magnification) and Golgi-stained hippocampal CA3 neurons (4-6) from control $(1,4)$, nonresponder $(2,5)$, and responder $(3,6)$ animals. Most of the BrdU-labeled cells are located in the subgranular zone (SGZ), the area between the $\mathrm{GCL}$ and the hilus $(\mathrm{H})$ in the hippocampus. Data presented as box plots show the 25 th and 75 th percentiles. The bars in the boxes represent the medians. ${ }^{*} p<0.05$ versus $\left(\right.$ rats; ${ }^{\dagger} p<0.05$ versus NR.

animals had CMS but no CSD, and C animals had neither CSD nor CMS. All animals were evaluated for depression at D57. Serum corticosterone concentrations were twofold higher in CMS than in control animals, with no significant difference in adrenal gland weight (Fig. 1b). Other biochemical parameters like serum and hippocampal BDNF concentrations were not significantly different between CMS and C rats (Fig. 1c). At the behavioral level, immobility time in the FST and sweet water consumption were similar in CMS and C rats (Fig. 1d). There were no apparent morphological alterations in hippocampal volume, hippocampal cell proliferation, total apical and basal dendrite lengths of CA3 hippocampal neurons, number of intersections and dendritic spines of CA3 hippocampal neurons (Fig. $1 d$ ), or total dendrite lengths of amygdala neurons $(653.5 \pm 35.2$ vs $722.9 \pm 44.1 \mu \mathrm{m})$. Results of statistical analyses are presented in Table 1. Apart from the expected change in corticosterone (Bielajew et al., 2002), the CMS did not produce overt biochemical, anatomical, and behavioral alterations (with the set of parameters used here).
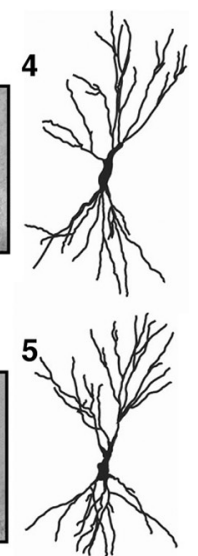

Interindividual variability at the end of the sensitization procedure

Striking differences were found in experimental animals subjected to the SSP, i.e., CSD followed by CMS (Fig. 2). Serum corticosterone concentration and immobility time measured in the FST in animals subjected to the SSP were significantly different from those measured in control rats or in CMS rats (Fig. 2). Serum BDNF concentration, sweet water consumption, and apical dendrite length of CA3 hippocampal neurons in animals subjected to the SSP were significantly different from those measured in control rats (Fig. 2). Hippocampal BDNF concentrations and hippocampal cell proliferation did not differ significantly between the total SSP population and control or CMS animals. Results of statistical analyses are presented in Table 2. All parameters measured in SSP animals were widely dispersed (Fig. 2). They appeared to include values with large modifications in addition to the values measured in CMS and C animals, suggesting that the SSP generated two populations of animals: one with alterations similar to CMS animals and one with large modifications. We thus tested the existence of two subgroups in experimental animals.

Identification of responder (depressive) and nonresponder (nondepressive) animal populations at the end of the SSP

Among all the biochemical, behavioral, and neuroanatomical parameters measured, statistical analysis revealed that serum corticosterone concentration was the most discriminative parameter for splitting different subpopulations (Wilk lambda, 0.29; partial lambda, $0.24 ; p=$ 0.0000001 ) (Figs. 3, 4). The key value for dividing the SSP animal population into two subpopulations was the highest value of serum corticosterone in CMS rats (43.94 $\mathrm{ng} / \mathrm{ml}$ ): those with values of $>43.94 \mathrm{ng} / \mathrm{ml}$, named "responder" (R) rats $(n=20)$ and those with values of $<43.94 \mathrm{ng} / \mathrm{ml}$, called "nonresponder" (NR) rats $(n=28)$ (Fig. $3 a)$. The increase of serum corticosterone concentration in $\mathrm{R}$ animals was associated with a higher adrenal gland weight $(+25 \%)$ (Fig. 3a). Responder rats had lower serum BDNF concentrations $(1.20 \pm 0.05 \mathrm{ng} / \mathrm{ml}$, mean \pm SEM $)$ than NR $(2.20 \pm 0.08 \mathrm{ng} / \mathrm{ml}$, mean \pm SEM $)$ or control animals $(2.16 \pm 0.11 \mathrm{ng} / \mathrm{ml}$, mean \pm SEM) (Fig. $3 b)$. Responder rats displayed depression-like behavior reflected by a significantly greater immobility time in the FST and significantly lower sweet water consumption than NR and C animals (Fig. 3c). Hippocampal BDNF concentrations in R animals (5.45 \pm 0.16 $\mathrm{pg} / \mathrm{mg}$, mean $\pm \mathrm{SEM}$ ) were significantly lower than those in NR $(7.94 \pm 0.30 \mathrm{pg} / \mathrm{mg}$ mean $\pm \mathrm{SEM})$ or C rats $(7.60 \pm 0.44 \mathrm{pg} / \mathrm{mg}$, mean \pm SEM) (Fig. 4a). The low hippocampal BDNF concentrations in $\mathrm{R}$ animals were associated with a lower hippocampal volume $(-18 \%)$, a smaller number of BrdU-positive hippocampal 
cells $(-20 \%)$, a retraction of apical CA3 hippocampal dendrites ( $-34 \%$; decreases of dendritic length and of dendritic intersection number), and spine density reduction (Fig. 4b,c), with no alteration of basal CA3 dendrite length (data not shown). Total dendrite lengths of amygdala neurons were significantly increased in $\mathrm{R}$ animals (C, $707.2 \pm 42.6 \mu \mathrm{m} ; \mathrm{NR}, 705.5 \pm 67.6 \mu \mathrm{m} ; \mathrm{R}$, $1039.6 \pm 37.4 \mu \mathrm{m})$. Results of statistical analyses are presented in Table 2. Thus, the $\mathrm{R}$ group presents biochemical, morphological, and behavioral traits that are very different from the NR group. Since CMS animals do not display these alterations, we hypothesized that they were triggered by the CSD protocol and that they persisted after CSD. To test this hypothesis, we performed a longitudinal analysis and tried to identify predictive biological markers of depression sensitivity.

\section{Identification of vulnerable and nonvulnerable animals before the CMS protocol corresponding to responder (depressive) and nonresponder (nondepressive) animals at the end of SSP, respectively}

At D9, all DF rats presented hypothalamicpituitary-adrenal (HPA) axis hyperactivity (increases of corticosterone concentrations and of adrenal gland weight), decreased BDNF concentration in serum and hippocampus, reduced hippocampal volume and neurogenesis and CA3 dendritic retraction (decreases of dendritic length and of dendritic intersection number), and spine density reduction as shown by comparison with control rats (animals not subjected to social defeat) (Figs. $5 a-d, 6,7)$. Total dendrite lengths of amygdala neurons were significantly increased in all defeated animals $(C$, $756.5 \pm 59.7 \mu \mathrm{m} ; \mathrm{DF}, 1141.7 \pm 44.7 \mu \mathrm{m})$. The drastic differences with controls as well as the homogeneity of the measured parameters in defeated rats did not allow identification of subpopulations in the latter pool of animals. In contrast, immobility time in the FST did not differ significantly between defeated and control animals ( $409.6 \pm 2.85$ vs $412.33 \pm 2.45 \mathrm{~s} ; n=15)$. We conclude that biochemical and morphological alterations are present soon after CSD. We then tested whether they were persistent.

At D35 (i.e., at the end of recovery period following CSD and before CMS), serum corticosterone concentrations, adrenal gland weight, and serum BDNF concentrations in defeated rats were not significantly different from those measured in control rats. However, contrary to serum corticosterone concentrations, values of serum BDNF concentrations in DF rats were highly heterogeneous (from 0.66 to $3.06 \mathrm{ng} / \mathrm{ml}$ ), with a set of values overlapping $\mathrm{C}$ rats and a set of high values, suggesting two subpopulations (Fig. 6a). To identify these subpopulations, a cluster analysis and a correlation between the most discriminative parameter at D35 (serum BDNF concentration; Wilk lambda, 0.63; partial lambda, $0.57 ; p=0.000025$ ) and the most discriminative parameter at D57 (serum corticosterone concentration; see above) were calculated in same animals (Fig. 6b). In light of the cluster analysis results and the significant negative correlation

\section{DAY 9}
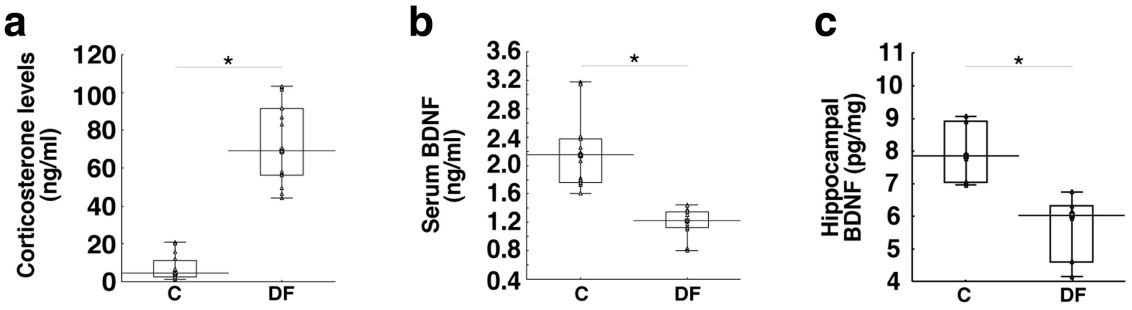

Adrenal gland weight:

C: $10.28 \pm 0.31 \mathrm{mg} / 100 \mathrm{~g}$

d Hippocampal volume: (n) Cis dendrite length, and number of dendritic spines. Sholl analysis for C and DF animals is shown. Data presented as box plots show the
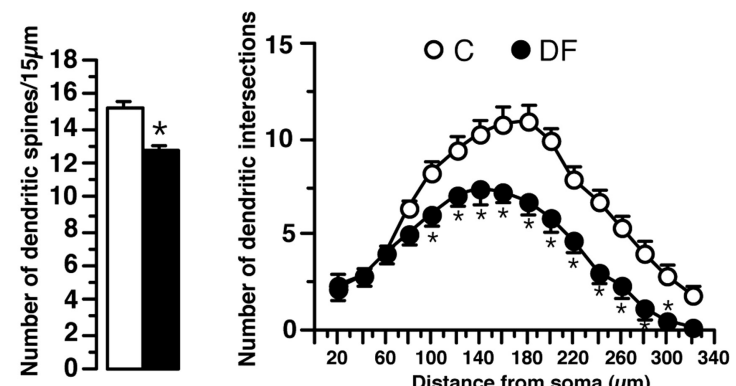

Distance from soma $(\mu \mathrm{m})$ 
a

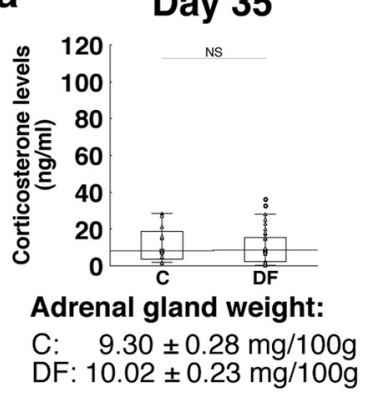

b

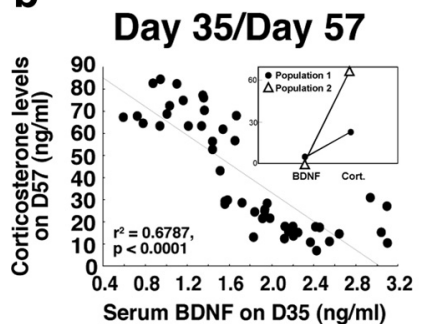

d

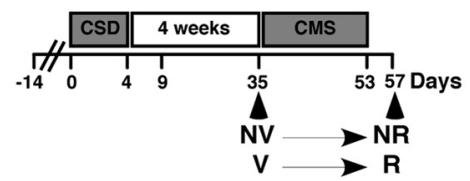

Figure 6. Identification of vulnerable and nonvulnerable animals. $\boldsymbol{a}$, At D35, determination of corticosterone concentrations, adrenal gland weight, and serum BDNF concentrations in defeated rats ( $n=37$ ) and in control rats (animals not subjected to social defeat; $n=12$ ). $\boldsymbol{b}$, Negative correlation between serum BDNF concentrations at D35 and the corticosterone concentrations of SSP rats at D57 in same animals ( $n=48$ ). Inset, Cluster analysis. $c$, Based on the negative correlation, rats were separated in two categories: nonvulnerable $(n=28)$ with circulating BDNF concentrations similar as those obtained in control rats $(n=15)$, and vulnerable $(n=20)$ animals with lower serum BDNF concentrations. $\boldsymbol{d}$, Longitudinal follow-up showed that the rats defined as NV or V animals at D35 became nondepressive (NR) and depressive (R), respectively, at the end of the SSP (D57). Data presented as box plots show the 25 th and 75 th percentiles. The bars in the boxes represent the medians. Circles represent atypical values. ${ }^{*} p<0.05$ versus $\left(\right.$ rats; ${ }^{\dagger} p<0.05$ versus NV.

logical alterations seen at D9 following CSD disappear in the NV group at D35, while they persist in the $\mathrm{V}$ group. Low levels of serum BDNF sign vulnerable animals.

Since depression appears in animals with reorganized hippocampal and amygdala circuits, a causal link may exist between both observations. Since BDNF is crucial for maintaining circuit integrity (Castrén and Rantamäki, 2010), low cortical BDNF levels may be causally related to the reorganization of the neuronal circuitry, hence the appearance of depression. If this scheme is correct, we reasoned that chronic central treatment with a BDNF agonist would restore circuit integrity and prevent depression in the $\mathrm{V}$ group.

\section{Effects of intracerebroventricular chronic treatment with 7,8-DHF on behavioral, biochemical, and neuroanatomical parameters}

Animals were identified as nonvulnerable (serum BDNF, $1.94 \pm$ $0.16 \mathrm{ng} / \mathrm{ml}$ ) or vulnerable (serum BDNF, $1.10 \pm 0.06 \mathrm{ng} / \mathrm{ml}$ ) at D35 and then treated with a central administration of 7,8-DHF (a TrkB receptor agonist) or vehicle (Fig. 8). Hippocampal parameters (volume, neurogenesis, apical dendritic length, intersections, and dendritic spines) measured at D57 were similar or were slight increased in 7,8-DHF-treated compared to vehicle-treated animals in both NV and control groups (Fig. 8a). Dendrite length in amygdala was similar in nonvulnerable animals treated with
7,8-DHF or vehicle as well as in treated or nontreated control animals $(759.03 \pm 33.78,695.80 \pm 29.18,700.22 \pm 29.60$, and $711.61 \pm 38.69 \mu \mathrm{m}$, respectively).

In contrast, in rats identified as vulnerable at D35, 7,8-DHF treatment completely prevented both hippocampal alterations measured at D57 compared to vulnerable rats receiving vehicle (Fig. $8 a$ ) and the increase in amygdala dendritic length $(1102.05 \pm 51.46$ vs $763.27 \pm 33.25 \mu \mathrm{m})$. More importantly, 7,8 -DHF treatment completely prevented the occurrence of depressive profile (lack of HPA hyperactivity, anhedonia, or helplessness behavior) (Fig. 8b). Results of statistical analyses are presented in Table 3. In conclusion, the brain infusion of BDNF receptor agonist prevented the vulnerable animals from becoming responder rats after the CMS protocol, strongly suggesting causality between sustained decreased BDNF levels and morphological alterations leading to sensitivity to depression. We then tested whether treatment with the antidepressant imipramine had similar positive effects.

Effects of chronic treatment with imipramine on behavioral, biochemical, and neuroanatomical parameters

Imipramine treatment started soon after CSD (Fig. 9). At D35, serum BDNF concentrations were not significantly different between imipramine-treated defeated (DI) animals (from 1.62 to $3.30 \mathrm{ng} / \mathrm{ml}$ ) and imipramine-treated control (CI) rats (from 1.71 to $2.53 \mathrm{ng} / \mathrm{ml}$ ). Thus, imipramine treatment decreased serum BDNF variability. As the data obtained in DI rats were similar to those measured in vehicle-treated NV animals, none of the DI rats could be classified as V or NV rats at D35 (Fig. 9a). At D57, serum BDNF concentrations in DI rats were not significantly different from those measured in vehicle-treated $\mathrm{C}$ rats, CI rats, or vehicle-treated NR animals (Fig. 9b). At D57, corticosterone concentrations in DI rats were twice those in CI animals and were not significantly different from those measured in vehicle-treated NR animals, whereas no significant difference in adrenal gland weight was observed between these groups (Fig. 9b). Imipramine treatment having been stopped before the start of the CMS protocol, this mild hyperactivity of the HPA axis is similar to that induced by 3 weeks of CMS alone. At D57, in DI animals, hippocampal BDNF, hippocampal volume, neurogenesis, the apical dendrite lengths of CA3 hippocampal neurons, spine density, and total dendrite lengths of amygdala neurons (718.2 \pm 32.8 vs $801.7 \pm 48.1 \mu \mathrm{m})$ did not significantly differ with vehicle-treated C rats, CI rats, or vehicle-treated NR animals, nor did immobility time in the FST (Fig. 9c). As all values of the different parameters were close to those measured in vehicle-treated NR animals and significantly different from those measured in vehicle-treated $\mathrm{R}$ animals, no DI rats could be classified as R rats at D57. Results of statistical analyses are presented in Table 4 .

\section{Discussion}

We performed a longitudinal study to determine the latent traits of vulnerability leading to the development of depressive disorders. At the end of SSP, all the measured parameters (biochemical, behavioral, and neuroanatomical) in SSP rats were highly heterogeneous, suggesting two animal subpopulations. Based on the statistical analysis showing that corticosterone was the most discriminative parameter and on the classification matrix that validated the classification in $100 \%$ of cases (Table 5), we identified one population ( $58 \%$ of all SSP animals), classified as nonresponders, with corticosterone concentrations of $<43.94 \mathrm{ng} / \mathrm{ml}$ and serum BDNF concentrations, immobility time in the FST, and sucrose preferences similar to those measured in control or 
CMS rats. The other population $(42 \%$ of all SSP animals), presenting a depressive phenotype characterized by HPA axis hyperactivity, low serum BDNF concentrations, despair behaviors, and anhedonia, was classified as responders. In $\mathrm{R}$ animals, the decrease in hippocampal BDNF concentrations associated with hippocampal morphological changes is consistent with the depressive phenotype described in humans (Sheline et al., 1999; Bremner et al., 2000; Duman and Monteggia, 2006; Pittenger and Duman, 2008). Animal models of depression and psychosocial stress are associated with reduced hippocampal neurogenesis, hippocampal dendritic arborization, and hippocampal BDNF that are prevented by antidepressant treatment (Watanabe et al., 1992; Magariños et al., 1996, 2011; Sapolsky, 2000; Czeh et al., 2001; Buwalda et al., 2005; Yap et al., 2006; Castrén et al., 2007; Czeh and Lucassen, 2007; Becker et al., 2008; Krishnan and Nestler, 2008; Alleva and Francia, 2009; Covington et al., 2009). Depending on studies, the hippocampal spine density is or not affected by stress events (Chen et al., 2010; Magariños et al., 2011). Concerning serum BDNF levels, published data lead to contradictory conclusions (Schulte-Herbrüggen et al., 2009; O'Mahony et al., 2011). There-

fore, our data show that $\mathrm{R}$ animals have been sensitized by social defeat. The data presented here show, for the first time, that rats with a depressive profile present a decline in hippocampal volume-resulting from decreases in hippocampal neurogenesis and the length of the dendrites of apical CA3 neurons - related to hippocampal BDNF decrease. Importantly, decrease in serum BDNF concentration reflects these changes. These hippocampal morphological alterations are accompanied by a hypertrophy of amygdala neurons as described in depressive patients (Pittenger and Duman, 2008). Therefore, we demonstrate the existence of two animal populations differing in their responses to the depressive sensitization procedure. Comparisons between R (depressive) and NR (nondepressive) animals thus constitute an ideal experimental approach for assessing neuroanatomical substrates underlying vulnerability state of depression.

This raises the question of whether morphological changes are specific to depression or whether they are present before its occurrence, thus constituting the residual traces underlying vulnerability to the emergence further depression. To address this question, it is crucial to determine whether all measured parameters were present before the start of the CMS protocol. Five days after the end of the chronic social-defeat procedure (D9), it was not possible to distinguish the two distinct subpopulations. Indeed, discrimination in two subpopulations was impossible as all defeated animals displayed marked changes in all measured parameters. However, these features were not correlated with a change of immobility time in the FST. Thus, the neuroanatomical alterations observed resulted from the chronic stress-related events and were not linked to depression-like behavior. These data strongly suggest that the decreased serum BDNF concentration and hippocampal remodelling cannot be considered specific markers of depression. In the short term, immediately after a b

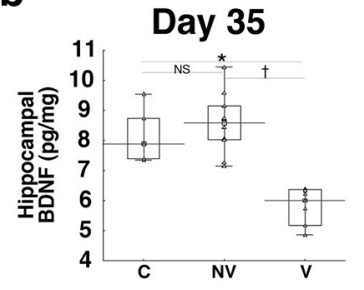

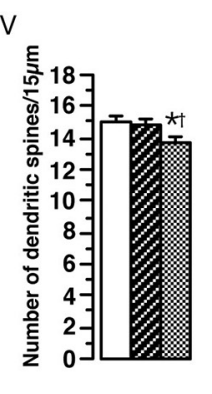

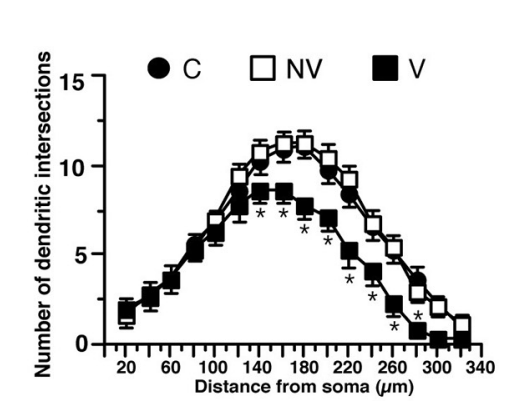

mpal volume:
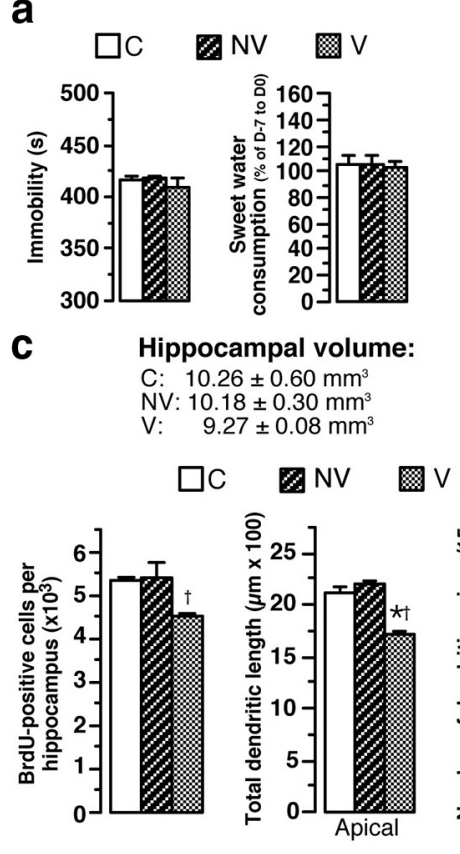

Figure 7. Behavioral, biochemical, and neuroanatomical parameters in vulnerable and nonvulnerable animals. a, At D35, NV, and C rats $(n=4-10)$. Sholl analysis in V, NV, and C animals is shown. Data presented as box plots show the 25 th and 75 th percentiles. The bars in the boxes represent the medians. ${ }^{*} p<0.05$ versus $C$ rats; ${ }^{\dagger} p<0.05$ versus NV.

chronic stress event, they appear more likely to act as a compensatory response, protecting against deleterious chronic stressrelated events, rather than as a maladaptive response (Conrad, 2006; McEwen, 2010).

Several weeks after CSD, we identified one population with biochemical, behavioral, and neuroanatomical parameters similar to those in control animals, whereas the other population displayed sustained lower levels of serum and hippocampal BDNF; reduced neurogenesis, apical CA3 dendrite lengths, spine density; and a hypertrophy of amygdala neurons. Since these animals were distinguishable from responder rats (D57) by the lack of depressive profile and a normal HPA axis activity, this subpopulation was defined as vulnerable. Vulnerable and nonvulnerable groups may correspond to the "unsusceptible" and "susceptible" groups (Krishnan et al., 2007). Importantly, our experimental paradigm is the first to define the outcome of these two populations after a second stress: nonvulnerable rats remain nondepressive (NR), whereas vulnerable rats become depressive (R). We can make use of this relevant experimental situation to identify predictive markers and mechanisms underlying this state of vulnerability. BDNF concentration is a good candidate marker for identifying animals as nonvulnerable if concentrations exceed $1.65 \mathrm{ng} / \mathrm{ml}$ ( 24 of $28 ; 86 \%$ ), and as vulnerable when concentrations are below $1.55 \mathrm{ng} / \mathrm{ml}$ (17 of 20;85\%). The longitudinal follow-up of our animals clearly shows that in the absence of depressive behavior, low BDNF concentrations associated with high corticosterone concentrations are specific of the state induced by the priming intense stress, and when associated with normal corticosterone concentrations, are specific of a state of vulnerability to depression. The data presented here demonstrate that, in some animals subjected to chronic stress, CA3 dendritic retraction is reversible consistent with the transient neuroprotec- 

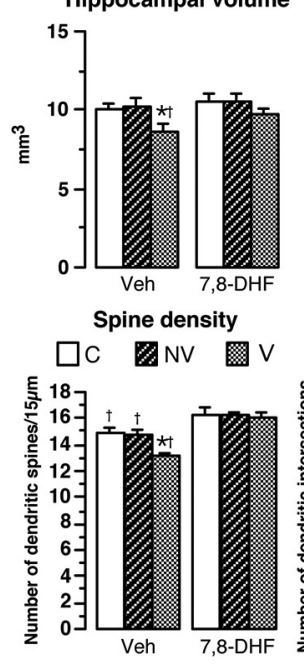

b $\square \mathrm{C}$ खNV $\mathrm{NV}$

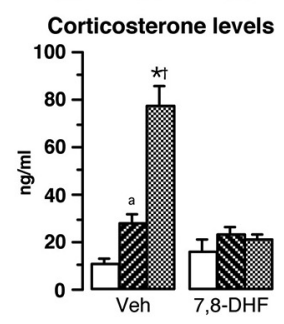

\section{HC}

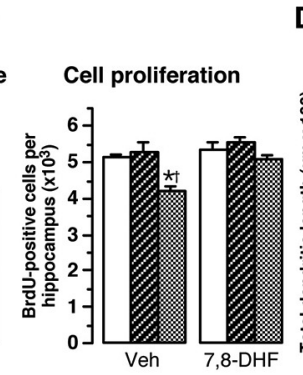

DAY 57

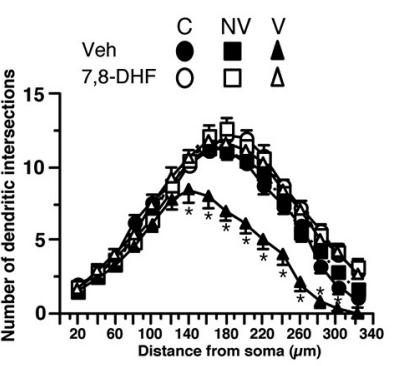

\section{Apical dendritic length}
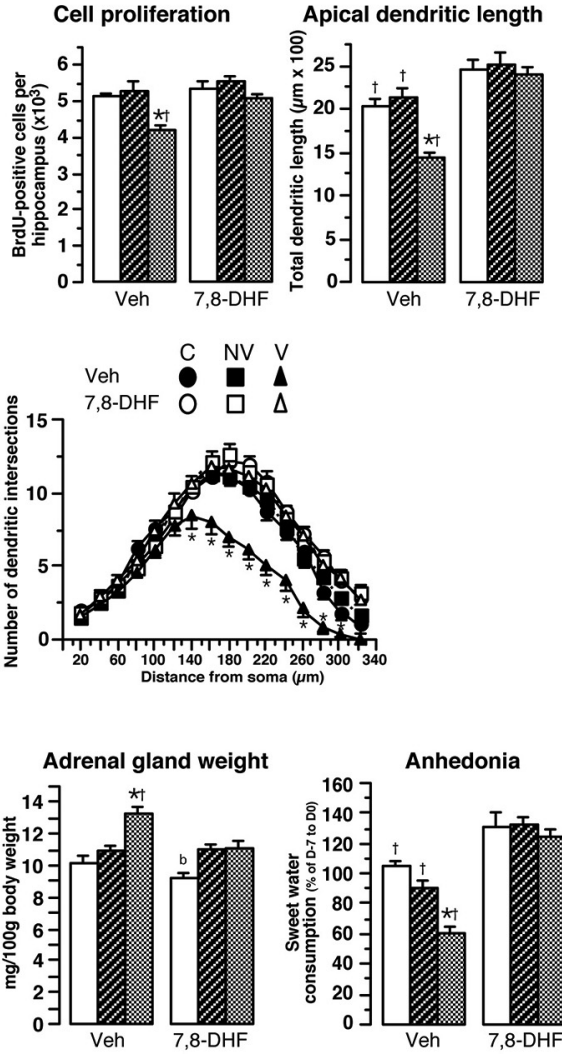

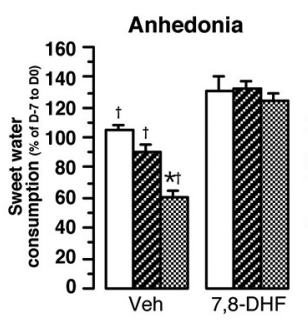

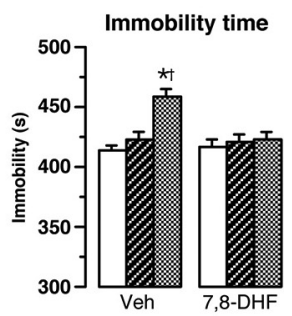

Figure 8. Chronic intracerebroventricular treatment with 7,8-DHF prevents biochemical, neuroanatomical, and behavioral parameters. a, 7,8-DHF was chronically delivered through ALZET minipumps from D35 until the end of the experiment (D57) in the brains of animals identified as nonvulnerable or vulnerable. In V rats, 7,8-DHF treatment reversed the decrease of hippocampal volume, neurogenesis, total apical dendrite lengths of CA3 hippocampal neurons, and spine density measured at the end of the treatment (D57; $n=4-7$ ) compared to vehicle-treated $V$ rats. Sholl analysis for vehicle-treated or 7,8-DHF-treated R, NR, and C animals is shown. $\boldsymbol{b}$, At D57, the increase of corticosterone levels and adrenal gland weight as well as the decrease of sweet water consumption (anhedonia) and the enhancement of immobility time in FST observed in identified $V$ animals treated with vehicle were completely prevented by chronic 7,8-DHF treatment $(n=8-13) .{ }^{*} p<0.05$ versus NV rats receiving vehicle; ${ }^{\dagger} p<0.05$ versus corresponding 7,8-DHF-treated groups; ${ }^{a} p<0.05$ versus $C$ rats receiving vehicle; ${ }^{b}<$ versus corresponding 7,8-DHFtreated vulnerable rats.

tive role attributed to CA3 dendritic remodelling (Conrad, 2006). The persistence of this neuronal alteration results from a low hippocampal BDNF level since chronic intracerebroventricular treatment with a selective TrkB receptor agonist (7,8-DHF; Jang et al., 2010) completely prevents it. Until now, it was established that BDNF signaling plays an important role in the behavioral and cellular efficacy of antidepressants, but the role of BDNF in the pathophysiology of depression is less clear (Castrén and Rantamäki, 2010). Our study demonstrates that BDNF decrease precedes the emergence of the disease. Thus, BDNF controls neuroanatomical alterations that constitute residual traces of the previous stress acting as factors of vulnerability to future stress. Indeed, chronic imipramine treatment during the recovery period completely blunted the biological characteristics of vulnerable animals, thus precluding the possibility to discriminate between vulnerable and nonvulnerable populations. Importantly, none of these imipramine-treated animals developed the "depressive" phenotype after the second mild stress. These observations strongly suggest that the changes associated with neural plasticity are related to a state of vulnerability to depressive disorders rather than to the depressive state itself. Such a statement is supported by the efficacy of chronic 7,8-DHF or imipramine treatment that completely suppressed residual traces responsible for sensitization to subsequent stressors and then prevents the development of depressive disorders.

This preclinical study is the first to show that stress can trigger biological and morphological changes in brain plasticity that induce depressive phenotype only when a second stressful event occurs. Our study clearly points out the key role of hippocampal and amygdala remodelling on depression, opening new research avenues to better understanding the pathophysiology of the disease. The existence of nonvulnerable and vulnerable animals clearly shows that changes in the structures of the hippocampus and amygdala constitute latent traits responsible for vulnerability to depression. Preventing the neuroanatomical alterations by 7,8-DHF intracerebroventricular administration demonstrates the involvement of the latent vulnerability traits in the development of depression-related behavior. Moreover, the strong correlation between decreases in serum BDNF concentration and changes in neuroplasticity strongly suggests that serum BDNF concentration reflects the presence of residual traces responsible for vulnerability to depression. Although the mechanisms allowing us to explain the relationship between serum and hippocampal BDNF are unknown, a recent study showed that measuring serum BDNF concentration can be used to monitor changes in neurotrophin levels in brain tissue (Sartorius et al., 2009). A decrease in serum BDNF concentration associated with normal serum cortisol concentration may be considered a relevant biomarker for identifying, within a population at risk, individuals more likely to develop depressive disorders. Thus, based on these data, it should be possible to identify high-risk individuals early, before the occurrence of other life events leading to the development of depressive disorders. Our results provide some bases not only for early diagnosis, but also for treatment. Indeed, the results obtained when brain morphological changes were prevented suggest that all treatments, even nonpharmacological treatments, suppressing residual traces in the hippocampus and the amygdala should also suppress vulnerability to the development of depressive disorders. Such changes may be extended to other diseases in which life stressor events predispose individuals to develop, for instance, epilepsy (Koe et al., 2009) or schizophrenia (Cannon et al., 2003).

\section{References}

Alleva E, Francia N (2009) Psychiatric vulnerability: suggestions from animal models and role of neurotrophins. Neurosci Biobehav Rev 33:525-536.

Andre J, Zeau B, Pohl M, Cesselin F, Benoliel JJ, Becker C (2005) Involvement of cholecystokininergic systems in anxiety-induced hyperalgesia in male rats: behavioral and biochemical studies. J Neurosci 25:7896-7904.

Becker C, Thiebot MH, Touitou Y, Hamon M, Cesselin F, Benoliel JJ (2001) Enhanced cortical extracellular levels of cholecystokinin-like material in a model of anticipation of social defeat in the rat. J Neurosci 21:262-269. 
Table 3. Statistical analyses of the stress effects and of 7,8-DHF treatment on biological, behavioral, and neuroanatomical parameters

\begin{tabular}{llll}
\hline & Stress effect & Treatment effect & Stress $\times$ treatment interactions \\
\hline Corticosterone levels & $F_{(2,57)}=17.89, p<0.0001$ & $F_{(1,57)}=20.98, p<0.0001$ & $F_{(2,57)}=13.84, p<0.0001$ \\
Adrenal gland weight & $F_{(2,57)}=16.74, p<0.0001$ & $F_{(1,57)}=9.19, p=0.003$ & $F_{(2,55)}=4.20, p=0.01$ \\
Cell proliferation & $F_{(2,20)}=11.40, p=0.0005$ & $F_{(1,20)}=6.29, p=0.02$ & $F_{(2,20)}=0.78, p=0.46$ \\
Hippocampal volume & $F_{(2,20)}=7.43, p=0.003$ & $F_{(1,20)}=7.04, p=0.01$ & $F_{(2,20)}=0.88, p=0.42$ \\
Apical dendrite length & $F_{(2,99)}=9.47, p<0.0001$ & $F_{(1,99)}=41.43, p<0.0001$ & $F_{(2,99)}=6.29, p=0.0027$ \\
Dendritic spines & $F_{(2,353)}=6.79, p=0.0013$ & $F_{(1,353)}=49.92, p<0.0001$ & $F_{(2,35)}=4.60, p=0.01$ \\
Dendrite length of amygdala neurons & $F_{(2,52)}=23.48, p<0.0001$ & $F_{(1,52)}=10.68, p=0.0019$ & $F_{(2,52)}=17.16, p<0.0001$ \\
Immobility time & $F_{(2,44)}=9.17, p=0.0005$ & $F_{(1,44)}=4.20, p=0.04$ & $F_{(2,44)}=6.63, p=0.003$ \\
Anhedonia & $F_{(2,57)}=10.20, p=0.0002$ & $F_{(1,57)}=3.09, p<0.0001$ & $F_{(2,57)}=4.96, p=0.01$ \\
\hline
\end{tabular}

Statistical analyses of the effects of stress and of 7,8-DHF treatment on hippocampal volume, cell proliferation, apical dendrite length of CA3 neurons, hippocampal CA3 dendritic spine, dendrite length of amygdala neurons, corticosterone levels, adrenal gland weight, sweet water consumption and immobility time, based on two-way ANOVA.

Becker C, Zeau B, Rivat C, Blugeot A, Hamon M, Benoliel JJ (2008) Repeated social defeatinduced depression-like behavioral and biological alterations in rats: involvement of cholecystokinin. Mol Psychiatry 13:10791092.

Bielajew C, Konkle AT, Merali Z (2002) The effects of chronic mild stress on male SpragueDawley and Long Evans rats: I. Biochemical and physiological analyses. Behav Brain Res 136:583-592.

Bremner JD, Narayan M, Anderson ER, Staib LH, Miller HL, Charney DS (2000) Hippocampal volume reduction in major depression. Am J Psychiatry 157:115-118.

Brown GW, Bifulco A, Harris TO (1987) Life events, vulnerability and onset of depression: some refinements. Br J Psychiatry 150:30-42.

Buwalda B, Kole MH, Veenema AH, Huininga M, de Boer SF, Korte SM, Koolhaas JM (2005) Long-term effects of social stress on brain and behavior: a focus on hippocampal functioning. Neurosci Biobehav Rev 29:83-97.

Cannon TD, van Erp TG, Bearden CE, Loewy R, Thompson P, Toga AW, Huttunen MO, Keshavan MS, Seidman LJ, Tsuang MT (2003) Early and late neurodevelopmental influences in the prodrome to schizophrenia: contributions of genes, environment, and their interactions. Schizophr Bull 29:653-669.

Castrén E, Rantamäki T (2010) The role of BDNF and its receptors in depression and antidepressant drug action: Reactivation of developmental plasticity. Dev Neurobiol 70:289-297.

Castrén E, Võikar V, Rantamäki T (2007) Role of neurotrophic factors in depression. Curr Opin Pharmacol 7:18-21.

Chen Y, Rex CS, Rice CJ, Dubé CM, Gall CM, Lynch G, Baram TZ (2010) Correlated memory defects and hippocampal dendritic spine loss after acute stress involve corticotropinreleasing hormone signaling. Proc Natl Acad Sci U S A 107:13123-13128.

Conrad CD (2006) What is the functional significance of chronic stress-induced CA3 dendritic retraction within the hippocampus? Behav Cogn Neurosci Rev 5:41-60.

Conrad CD, McLaughlin KJ, Harman JS, Foltz C, Wieczorek L, Lightner E, Wright RL (2007) Chronic glucocorticoids increase hippocampal vulnerability to neurotoxicity under conditions that produce CA3 dendritic retraction but fail to impair spatial recognition memory. J Neurosci 27:8278-8285.

Covington HE III, Vialou V, Nestler EJ (2009) From synapse to nucleus: Novel targets for treating depression. Neuropharmacology 58:683-693.

Cryan JF, Slattery DA (2007) Animal models of mood disorders: Recent developments. Curr Opin Psychiatry 20:1-7.

Czeh B, Lucassen PJ (2007) What causes the hippocampal volume decrease

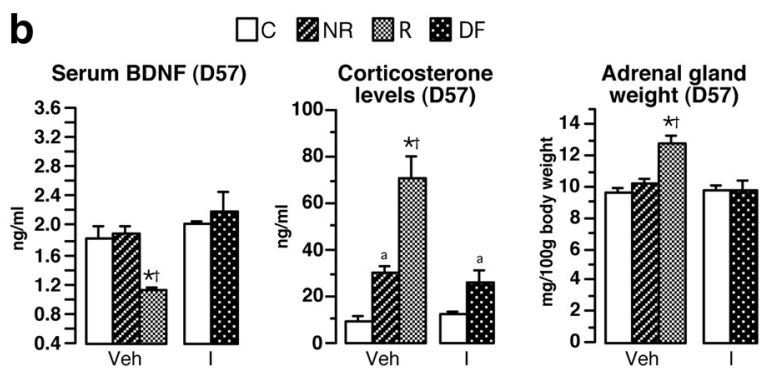

Cell proliferation (D57) Apical dendritic length (D57)

Hippocampal volume: C: $9.98 \pm 0.35 \mathrm{~mm}^{3}$ $\mathrm{NR}: 10.11 \pm 0.43 \mathrm{~mm}^{3}$ R: $\quad 8.28 \pm 0.46 \mathrm{~mm}^{3}$ * Cl: $10.36 \pm 0.65 \mathrm{~mm}^{3}$ DI: $10.44 \pm 0.37 \mathrm{~mm}^{3}$
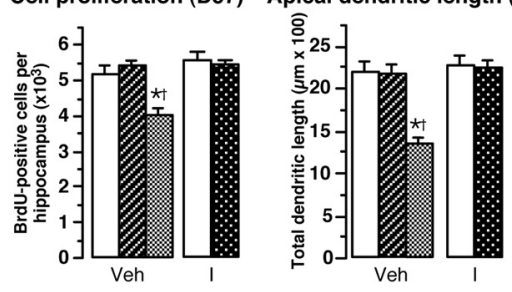

Immobility time (D57)

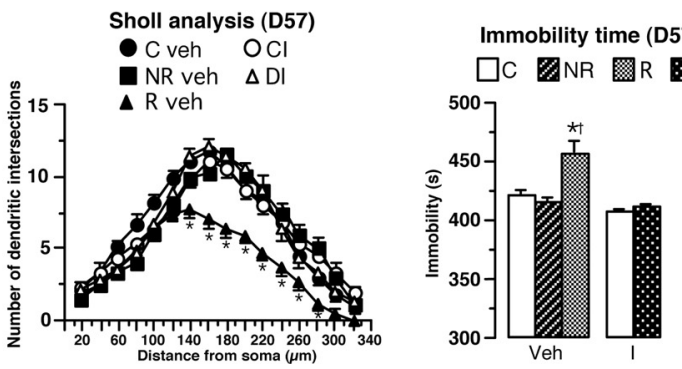

Figure 9. Chronic treatment with imipramine reverses biochemical, neuroanatomical, and behavioral parameters. $\boldsymbol{a}$, Imipramine (16 $\mathrm{mg} / \mathrm{kg} / \mathrm{d}$ ) were delivered through ALZET osmotic minipumps, implanted subcutaneously on the backs of animals. Serum BDNF concentrations at D35 in imipramine-treated and vehicle-treated control rats, imipramine-defeated animals, and defeated rats receiving vehicle (nonvulnerable and vulnerable; $n=11-13$ ) are shown. $\boldsymbol{b}$, At D57, serum BDNF concentration, corticosterone levels, and adrenal gland weight in imipramine-treated and vehicle-treated control rats, imipramine-defeated animals, and defeated rats receiving vehicle (nonredendrite length of $C A 3$ hippocampal neurons, spine density, Sholl analysis, and immobility time in the FST in defeated rats receiving vehicle (NR, R; $n=4-15) .{ }^{*} p<0.05$ versus NV animals receiving vehicle; ${ }^{\dagger} p<0.05$ versus $\mathrm{DI} ;{ }^{\mathrm{a}} p<0.05$ versus corresponding ( groups.

in depression? Are neurogenesis, glial changes and apoptosis implicated? Eur Arch Psychiatry Clin Neurosci 257:250-260.

Czeh B, Michaelis T, Watanabe T, Frahm J, de Biurrun G, van Kampen M, Bartolomucci A, Fuchs E (2001) Stress-induced changes in cerebral metabolites, hippocampal volume, and cell proliferation are prevented by antidepressant treatment with tianeptine. Proc Natl Acad Sci U S A 98:12796-12801.

de Kloet ER, Joels M, Holsboer F (2005) Stress and the brain: from adaptation to disease. Nat Rev Neurosci 6:463-475.

Duman RS, Monteggia LM (2006) A neurotrophic model for stress-related mood disorders. Biol Psychiatry 59:1116-1127.

Jabbi M, Kema IP, van der Pompe G, te Meerman GJ, Ormel J, den Boer JA 
Table 4. Statistical analyses of the stress effects and of imipramine treatment on biological, behavioral, and neuroanatomical parameters

\begin{tabular}{ll}
\hline & Stress effect \\
\hline Serum BDNF & \\
D35 & $F_{(4,61)}=7.66, p<0.0001$ \\
D57 & $F_{(4,61)}=6.15, p=0.0002$ \\
Corticosterone levels & $F_{(4,48)}=16.87, p<0.0001$ \\
Adrenal gland weight & $F_{(4,48)}=7.05, p<0.0001$ \\
Hippocampal BDNF levels & $F_{(4,20)}=6.71, p=0.001$ \\
Hippocampal volume & $F_{(4,17)}=3.94, p=0.018$ \\
Cell proliferation & $F_{(4,17)}=8.99, p=0.0004$ \\
Apical dendrite length & $F_{(4,67)}=26.35, p<0.0001$ \\
Dendritic spines & $F_{(4,218)}=11.18, p<0.0001$ \\
Dendrite length of amygdala neurons & $F_{(4,51)}=18.51, p<0.0001$ \\
Immobility time & $F_{(4,44)}=23.79, p<0.0001$ \\
\hline
\end{tabular}

Statistical analyses of the effects of stress and of imipramine treatment, on serum BDNF levels, corticosterone levels, adrenal gland weight, hippocampal BDNF concentration, hippocampal volume, cell proliferation, apical dendrite length of $C A 3$ neurons, hippocampal CA3 dendritic spine, dendrite length of amygdala neurons and immobility time, based on one-way ANOVA.

Table 5. Discriminant analysis on D57

\begin{tabular}{llcc}
\hline & \% Correct & NR, $p=0.58333$ & $\mathrm{R}, p=0.41667$ \\
\hline Nonresponder & 100 & 28 & 0 \\
Responder & 100 & 0 & 20 \\
Total & 100 & 28 & 20 \\
\hline
\end{tabular}

Classification matrix for D57 after discriminant analysis with Statistica is shown.

(2007) Catechol-o-methyltransferase polymorphism and susceptibility to major depressive disorder modulates psychological stress response. Psychiatr Genet 17:183-193.

Jacobson LH, Cryan JF (2007) Feeling strained? Influence of genetic background on depression-related behavior in mice: a review. Behav Genet 37:171-213.

Jang SW, Liu X, Yepes M, Shepherd KR, Miller GW, Liu Y, Wilson WD, Xiao G, Blanchi B, Sun YE, Ye K (2010) A selective TrkB agonist with potent neurotrophic activities by 7,8-dihydroxyflavone. Proc Natl Acad Sci U S A 107:2687-2692.

Karege F, Bondolfi G, Gervasoni N, Schwald M, Aubry JM, Bertschy G (2005) Low brain-derived neurotrophic factor (BDNF) levels in serum of depressed patients probably results from lowered platelet BDNF release unrelated to platelet reactivity. Biol Psychiatry 57:1068-1072.

Kessler RC (1997) The effects of stressful life events on depression. Annu Rev Psychol 48:191-214.

Kim YK, Lee HP, Won SD, Park EY, Lee HY, Lee BH, Lee SW, Yoon D, Han C, Kim DJ, Choi SH (2007) Low plasma BDNF is associated with suicidal behavior in major depression. Prog Neuropsychopharmacol Biol Psychiatry 31:78-85.

Koe AS, Jones NC, Salzberg MR (2009) Early life stress as an influence on limbic epilepsy: an hypothesis whose time has come? Front Behav Neurosci 3:1-16.

Krishnan V, Nestler EJ (2008) The molecular neurobiology of depression. Nature 455:894-902.

Krishnan V, Han MH, Graham DL, Berton O, Renthal W, Russo SJ, Laplant Q, Graham A, Lutter M, Lagace DC, Ghose S, Reister R, Tannous P, Green TA, Neve RL, Chakravarty S, Kumar A, Eisch AJ, Self DW, Lee FS, et al. (2007) Molecular adaptations underlying susceptibility and resistance to social defeat in brain reward regions. Cell 131:391-404.

Magariños AM, McEwen BS, Flügge G, Fuchs E (1996) Chronic psychosocial stress causes apical dendritic atrophy of hippocampal CA3 pyramidal neurons in subordinate tree shrews. J Neurosci 16:3534-3540.
Magariños AM, Li CJ, Toth JG, Bath KG, Jing D, Lee FS, McEwen BS (2011) Effect of brain-derived neurotrophic factor haploinsufficiency on stressinduced remodeling of hippocampal neurons. Hippocampus 21:253264.

McEwen BS (2010) Stress, sex, and neural adaptation to a changing environment: mechanisms of neuronal remodeling. Ann N Y Acad Sci 1204:E38-59.

McEwen BS, Stellar E (1993) Stress and the individual. Mechanisms leading to disease. Arch Intern Med 153:2093-2101.

McLaughlin KJ, Baran SE, Wright RL, Conrad CD (2005) Chronic stress enhances spatial memory in ovariectomized female rats despite CA3 dendritic retraction: possible involvement of CA1 neurons. Neuroscience 135:1045-1054.

Miczek KA, Yap JJ, Covington HE III (2008) Social stress, therapeutics and drug abuse: preclinical models of escalated and depressed intake. Pharmacol Ther 120:102-128.

O'Mahony CM, Clarke G, Gibney S, Dinan TG, Cryan JF (2011) Strain differences in the neurochemical response to chronic restraint stress in the rat: relevance to depression. Pharmacol Biochem Behav 97:690-699.

Paxinos P, Watson C (1986) The rat brain in stereotaxic coordinates, Ed 2. Sydney: Academic.

Pittenger C, Duman RS (2008) Stress, depression, and neuroplasticity: a convergence of mechanisms. Neuropsychopharmacology 33:88-109.

Post RM (1992) Transduction of psychosocial stress into the neurobiology of recurrent affective disorder. Am J Psychiatry 149:999-1010.

Sapolsky RM (2000) Glucocorticoids and hippocampal atrophy in neuropsychiatric disorders. Arch Gen Psych 57:925-935.

Sartorius A, Hellweg R, Litzke J, Vogt M, Dormann C, Vollmayr B, DankerHopfe H, Gass P (2009) Correlations and discrepancies between serum and brain tissue levels of neurotrophins after electroconvulsive treatment in rats. Pharmacopsychiatry 42:270-276

Schmidt HD, Duman RS (2010) Peripheral BDNF produces antidepressant-like effects in cellular and behavioral models. Neuropsychopharmacology 35:2378-2391.

Schulte-Herbrüggen O, Fuchs E, Abumaria N, Ziegler A, Danker-Hopfe H, Hiemke C, Hellweg R (2009) Effects of escitalopram on the regulation of brain-derived neurotrophic factor and nerve growth factor protein levels in a rat model of chronic stress. J Neurosci Res 87:2551-2560.

Sheline YI, Sanghavi M, Mintun MA, Gado MH (1999) Depression duration but not age predicts hippocampal volume loss in medically healthy women with recurrent major depression. J Neurosci 19:5034-5043.

Shimizu E, Hashimoto K, Okamura N, Koike K, Komatsu N, Kumakiri C, Nakazato M, Watanabe H, Shinoda N, Okada S, Iyo M (2003) Alterations of serum levels of brain-derived neurotrophic factor (BDNF) in depressed patients with or without antidepressants. Biol Psychiatry $54: 70-75$.

Sholl DA (1953) Dendritic organization in the neurons of the visual and motor cortices of the cat. J Anat 87:387-406.

Szapacs ME, Mathews TA, Tessarollo L, Ernest Lyons W, Mamounas LA, Andrews AM (2004) Exploring the relationship between serotonin and brain-derived neurotrophic factor: analysis of BDNF protein and extraneuronal 5-HT in mice with reduced serotonin transporter or BDNF expression. J Neurosci Methods 140:81-92.

Watanabe Y, Gould E, McEwen BS (1992) Stress induces atrophy of apical dendrites of hippocampal CA3 pyramidal neurons. Brain Res 588: 341-345.

Willner P, Muscat R, Papp M (1992) Chronic mild stress-induced anhedonia: a realistic animal model of depression. Neurosci Biobehav Rev 16:525-534

Yap JJ, Takase LF, Kochman LJ, Fornal CA, Miczek KA, Jacobs BL (2006) Repeated brief social defeat episodes in mice: Effects on cell proliferation in the dentate gyrus. Behav Brain Res 172:344-350. 